\title{
SOLUÇÃO NUMÉRICA PARA O PROCESSO DA INFILTRAÇÃO DA ÁGUA NO SOLO
}

\section{NUMERIC MODELING TO SIMULATE THE SOIL WATER INFILTRATION PROCESS}

\author{
Celso Luiz PREVEDELLO ${ }^{1}$ \\ Jocely Maria Thomazoni LOYOLA² \\ Marcos dos Santos COSTABILE ${ }^{3}$ \\ José HORODENSKI ${ }^{3}$
}

\begin{abstract}
RESUMO
Foram realizados vários ensaios laboratoriais para validar a hipótese de aderência entre os valores observados e simulados por um modelo numérico para o processo da infiltração da água no solo. Esse modelo foi desenvolvido a partir da linearização da equação de Richards no espaço e do uso da técnica iterativa de Newton-Raphson para resolver essa equação não linear no tempo. Os resultados mostraram que o modelo é capaz de simular o processo unidimensional da infiltração vertical da água no solo, tanto para solo homogêneo quanto estratificado.
\end{abstract}

Palavras-chave: Infiltração da água no solo, equação de Richards

\begin{abstract}
Several laboratorial tests were carried out with the objective to evaluate the statistical hypothesis of the equalities between observated and simulated values from a numerical model of the soil water infiltration process. This model was created Richards' equation linearized in space and by Newton-Raphson iterative process to solve this unlinearized equation on time. The results showed that model was reliable to evaluate the one-dimensional soil water infiltration process for both homogeneous and heterogeneous porous media.
\end{abstract}

Key-words: Soil water infiltration, Richard's equation

\footnotetext{
${ }^{1}$ Prof. Titular, Departamento de Solos da UFPR, clpreve@ufpr.br. Pesquisador do CNPq

${ }^{2}$ Aluno do programa de pós-graduação em Ciência do Solo da UFPR

${ }^{3}$ Aluno de graduação do Curso de Agronomia da UFPR.
} 
PREVEDELLO, C.L. et al. Solução numérica para o processo...

\section{INTRODUÇÃO}

Todos os processos dinâmicos da água no solo, como infiltração, redistribuição e evaporação, são tipicamente transientes e a solução analítica da equação diferencial parcial que descreve esses processos (equação de Richards), quando possível, resulta em grande grau de dificuldade. Isso, do ponto de vista matemático, decorre do fato da equação de Richards ser não linear, isto é, a condutividade hidráulica $\mathrm{K}$ na equação é função da variável dependente (umidade $\theta$ ou potencial mátrico da água no solo p/pg), que por sua vez é função da posição no espaço e do tempo. E a relação entre a condutividade hidráulica com a umidade (ou p/pg) é tal que a equação de Richards resulta altamente não linear. Uma equação assim, via de regra, só admite solução analítica quando linearizada. Mas nem sempre uma equação diferencial pode ser linearizada, a menos que erros até significativos sejam introduzidos (3). Modernamente, com o advento dos microcomputadores, as soluções desses problemas podem ser encontradas através de métodos numéricos, com grandes vantagens.

Este trabalho teve por objetivo testar experimentalmente a hipótese de validade de aderência entre os valores observados e simulados por um modelo numérico para o processo da infiltração da água no solo, desenvolvido a partir da linearização da equação de Richards no espaço e do uso da técnica iterativa de Newton-Raphson para resolver essa equação não linear no tempo.

\section{CONSIDERAÇÕES TEÓRICAS}

Com base na teoria desenvolvida por Mualem (5), nas equações combinadas de Van Genuchten (9) para as funções $\theta(\mathrm{p} / \rho \mathrm{g}), \mathrm{K}(\mathrm{p} / \rho \mathrm{g})$ (sendo $\mathrm{K}$ a condutividade, $\theta$ a umidade, e p/pg a pressão da água no solo), e no argumento de que a forma integrada da lei de Buckingham-Darcy pode produzir uma expressão analítica para a densidade de fluxo mátrico em regime de fluxo não transiente, então é possível linearizar a equação de Richards no espaço. Com isso, o que restará será uma equação não linear no tempo, cuja solução poderá ser obtida mediante uma técnica iterativa, como a de Newton-Raphson, por exemplo. As considerações que se seguem visam o desenvolvimento analítico deste postulado.

\section{PROPRIEDADES HIDRÁULICAS DO SOLO E SUAS FUNÇÕES COMBINADAS}

Desde que muitos dos parâmetros hidráulicos do solo são difíceis de ser obtidos em condições de campo, seja pela variabilidade a que estão sujeitos, seja pelo custo e/ou demanda de tempo dessas medidas, muitos autores têm procurado estabelecer, modificar ou substabelecer modelos para predizer a condutividade hidráulica como uma função da umidade a partir de outros parâmetros hidráulicos mais facilmente mensuráveis, como, por exemplo, da curva de retenção de água do solo e da condutividade hidráulica na saturação (5 e 9). A teoria desenvolvida por Mualem (5) mostra-se particularmente interessante porque permite a derivação analítica de uma série de importantes funções hidráulicas do solo, desde que a dependência $\mathrm{p} / \mathrm{\rho g}$ versus $\theta$ (curva de retenção de água no solo) também seja conhecida analiticamente. Um modelo empírico bastante versátil e que tem sido bastante utilizado $(4,6$ e 8) para representar analiticamente a curva de retenção de água no solo foi proposto por Van Genuchten (9), ou seja,

$$
\begin{array}{r}
\Theta=\frac{\theta-\theta_{r}}{\theta_{s}-\theta_{r}}=\frac{1}{\left[1+(\alpha|p / \rho g|)^{n}\right]^{m}} \\
\text { sendo } \mathrm{m}=1-1 / \mathrm{n}
\end{array}
$$

A equação (1), quando introduzida na teoria de Mualem (5), resulta que a condutividade hidráulica pode ser expressa como uma função de q ou p/ rg, através das seguintes expressões:

$$
K(\Theta)=K_{s} \Theta^{1 / 2}\left[1-\left(1-\Theta^{1 / m}\right)^{m}\right]^{2}
$$

sendo $\Theta=\frac{\theta-\theta_{r}}{\theta_{s}-\theta_{r}}$ ou $\Theta=\frac{1}{\left[1+(\alpha|p / \rho g|)^{n}\right]^{m}}$

em que a, n, $q_{s}$ (umidade na saturação), $q_{r}$ (umidade residual) e $m(m=1-1 / n)$ são parâmetros independentes de ajuste que devem ser estimados pelo ajuste da equação (1) aos dados observados da curva de retenção de água no solo. Os parâmetros independentes dessa equação $\left(a, n, m, q_{s}\right.$ e $\left.q_{r}\right)$ também podem ser simultaneamente conhecidos pela técnica dos mínimos quadrados para ajuste de funções não lineares (1). 


\section{SOLUÇÃO NUMÉRICA DA EQUAÇÃO DE RICHARDS}

Para expressar os processos unidirecionais da infiltração, redistribuição e evaporação da água do solo, Prevedello (7) obteve a seguinte expressão:

$$
\begin{aligned}
& \frac{\left(\theta_{i}^{j+1}-\theta_{i}^{j}\right)}{\Delta t}\left(z_{i}-z_{i-1}\right)=\left[\frac{K_{i+1} \cdot p_{i+1} / \rho g}{1-E E_{i+1}}-\frac{K_{i} \cdot p_{i} / \rho g}{1-E E_{i}}\right] /\left(z_{i+1}-z_{i}\right)- \\
& -K_{i}-\left[\frac{K_{i} \cdot p_{i} / \rho g}{1-E E_{i}}-\frac{K_{i-1} \cdot p_{i-1} / \rho g}{1-E E_{i-1}}\right] /\left(z_{i}-z_{i-1}\right)+K_{i-1}
\end{aligned}
$$$$
\text { sendo } E E=2+2,5 \mathrm{n} \cdot \mathrm{m}
$$

onde Dt é o intervalo de tempo em que ocorre a variação $q_{i}^{j+1}-q_{i}^{j}$, sendo $q_{i}^{j+1}$ e $q_{i}^{j}$, as umidades na profundidade $\mathrm{i}$ nos tempos $\mathrm{j}+1 \mathrm{e} \mathrm{j}$, respectivamente. O primeiro membro da (3) assume que a umidade na camada $\mathrm{z}_{\mathrm{i}}-\mathrm{Z}_{\mathrm{i}-1}$ é representada pela umidade da profundidade $\mathrm{i}$. Esse membro representa a variação de armazenamento de água ocorrida na camada $\mathrm{z}_{\mathrm{i}}-\mathrm{z}_{\mathrm{i}-1}$ durante o intervalo de tempo Dt. Num perfil de solo com i profundidades é necessário encontrar os valores de $\mathrm{p} / \mathrm{rg}$ que satisfazem a igualdade da (3) para assegurar o princípio da conservação da matéria em todas essas profundidades, desde $\mathrm{i}=1$ até $\mathrm{N}$, onde $\mathrm{N}$ é o número total de profundidades consideradas. $\mathrm{E}$ esses valores de $\mathrm{p} / \mathrm{rg}$ que asseguram a igualdade da (3) podem ser conhecidos, já que a função $K(p / r g)$ e $q(p / r g)$ são conhecidas (equação 2). Dessa forma, a equação de Richards, na qual a condutividade hidráulica é função da variável dependente (q ou p/rg), passa a ser linearizada no espaço. E o problema agora resulta resolver uma equação não linear no tempo. $E$ é nesse aspecto que o método iterativo de NewtonRaphson se apresenta particularmente interessante. Para aplicar esse método na (3) é necessário derivála com respeito a $p_{i} / \mathrm{rg}$, o que resulta:

$\frac{\alpha m\left(\theta_{s}-\theta_{r}\right) \Theta^{1 / m}\left(1-\Theta^{1 / m}\right)^{m}}{(1-m) \Delta t}\left(z_{i}-z_{i-1}\right)=\frac{K_{i}}{z_{i+1}-z_{i}}-\frac{E E_{i} K_{i}}{p_{i} / \rho g}+\frac{K_{i}}{z_{i}-z_{i-1}}$
As equações (3) e (4) devem ser resolvidas para se encontrar $\mathrm{p} / \mathrm{rg}$ no final de cada tempo, juntamente com as condições de fronteira. Essas condições, via de regra, são a definição de um fluxo ou de um valor de p/rg no tôpo ou no fundo do perfil (ou de uma coluna) de solo. Se existe lençol freático em $z=i$, por exemplo, há que se impor $\mathrm{p} / \mathrm{rg}=0$. Em caso contrário, $p_{i} / r g$ pode assumir um valor constante, previamente estimado ou admitido. No caso da infiltração

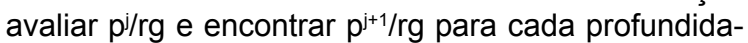
de de interesse no solo; e elas deverão ser novamente utilizadas para reavaliar esses termos quantas vezes forem necessárias (iterações) até que ocorra a convergência, ou seja, quando os dois membros da (3) se igualem, afim de se assegurar o princípio da conservação da matéria.

Para obter as equações (3) e (4), Prevedello (7) teve que adotar uma simplificação que se impôs pela necessidade de ter que integrar a equação (2). Acontece que a (2) é uma binomial extremamente complexa para ser integrada, gerando uma nova integral como parte da solução. Diante disso, Prevedello (7) constatou, após analisar as teorias de Van Genuchten (9) e Mualem (5), que a causa dessa complexidade residia no fator unitário no denominador do modelo de Van Genuchten (9) (equação 1). Prevedello (7), então, desprezou esse termo, implicando numa limitação de sua teoria em situações que não cumprem a condição $1 / 2 \mathrm{p} / \mathrm{rg}^{1} / 2>>1 / a$.

Com base nas equações (3) e (4), Prevedello (7) elaborou o programa abaixo, escrito em linguagem BASIC (Beginner's All-purpose Symbolic Instructional Code). Os principais passos utilizados na elaboração desse programas são comentados a seguir. O programa simula o processo de infiltração vertical da água em solo com uma determinada umidade inicial. Ele fornece os perfis de umidade e de pressão da água no solo para cada tempo de infiltração, bem como a taxa de infiltração instantânea na superfície. Os dados do solo são solicitados da linha 40 até 87 . Na linha 100 é solicitado o intervalo de tempo desejado entre os perfis de infiltração. As linhas 105 e 107 definem as profundidades do solo para as quais os valores de umidade e de pressão serão calculados. A linha 140 define os valores de umidade e de pressão no tempo inicial $(t=0)$ e a linha 227 calcula os respectivos valores de K. A equação (3) é calculada na linha 280 , para cada profundidade, e os valores da função derivada (equação 4), nas respectivas profundidades, são calculados na linha 270 . 
PREVEDELLO, C.L. et al. Solução numérica para o processo...

\section{PROGRAMA PARA SIMULAÇÃO DO PROCESSO DE INFILTRAÇÃO UNIDIRECIONAL DA ÁGUA NO SOLO}

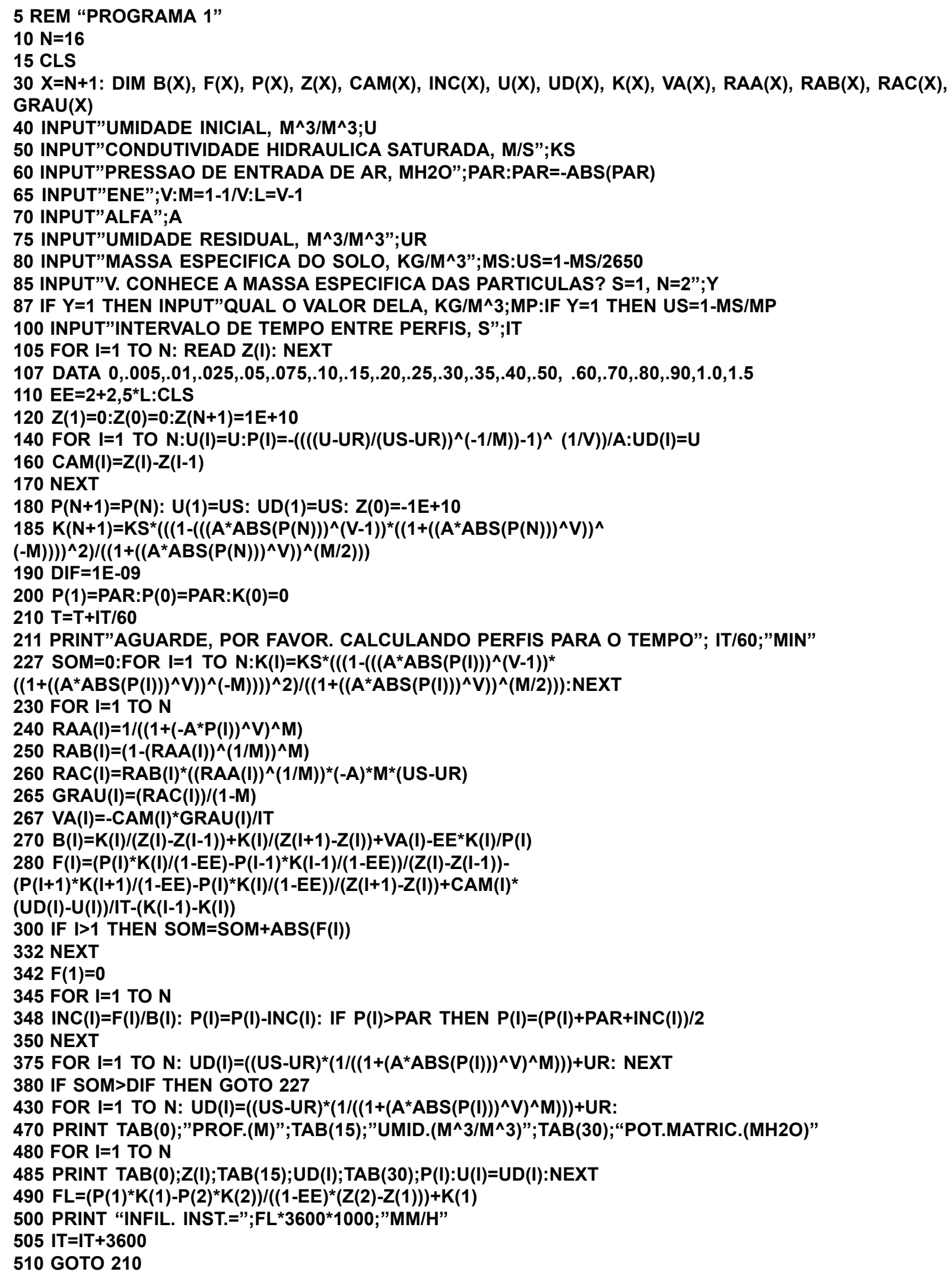


Na linha 300 são acumulados os valores das diferenças entre o primeiro e segundo membro da (3), para todas as profundidades consideradas. A 342 aplica uma condição de fronteira na superfície do solo. As linhas 345 a 350 calculam os incrementos de pressão que devem ser somados aos valores de pressão do último intervalo de tempo. Na 375 são calculados os valores de umidade do próximo perfil, através da equação (1). Se a soma acumulada dos valores das diferenças entre o primeiro e segundo membro da (3) de todas as profundidades não se anula dentro da precisão imposta na linha 190, então um novo cálculo é solicitado na linha 380 . Se nessa linha a condição de convergência for satisfeita, os resultados serão impressos pelo comando das linhas 460 a 485, bem como a taxa de infiltração instantânea, a qual é calculada nas linhas 490 e 500 . Finalmente, tudo se repete para o novo intervalo de tempo pelo comando da linha 510.

\section{METODOLOGIA}

\section{Avaliação experimentl do moelo numérico}

Para uma avaliação do modelo numérico apresentado no item anterior, foram realizados alguns ensaios laboratoriais envolvendo o processo unidimensional da infiltração da água do solo. Esses ensaios foram conduzidos em condições controladas no Laboratório de Física de Solos do Setor de Ciências Agrárias da UFPR. Foram utilizados dois materiais porosos distintos, do ponto de vista físico-químico: uma Areia Marinha, do balneário Caiobá, Município de Matinhos (PR), e um Latossolo vermelho-escuro, textura argilosa, do Município de Santa Terezinha de Itaipu (PR). A profundidade de coleta esteve entre 0 e $0,25 \mathrm{~m}$. Após secagem ao ar, ambos os materiais foram peneirados em malha de $2 \mathrm{~mm}$ e acondicionados em colunas de acrílico com 1,20m de altura e $50 \mathrm{~mm}$ de diâmetro interno. Para minimizar a segregação de partículas por ocasião do preenchimento das colunas, utilizou-se de um tubo extensor munido de um funil pelo qual o material poroso era continuamente depositado, de tal forma que o tubo ficasse permanentemente completo, evitando-se, dessa forma, que as partículas caíssem em queda livre. Foram montadas 3 colunas para cada tipo de material poroso. Em todas elas, quando verticalmente posicionadas, foram deixados $70 \mathrm{~mm}$ livres de solo na superfície, a fim de facilitar a manutenção de uma carga hidráulica de $50 \mathrm{~mm}$, a qual era obtida através de um frasco de Mariotte. Nas extremidades inferiores dessas colunas foram fixados tecidos de modo a sustentar o solo, sem, contudo, oferecer resistência ao fluxo.

Os parâmetros hidráulicos do solo foram obtidos a partir da curva de retenção de água por meio de mesa de tensão, empregando-se papel "mata bor- rão" de $1,0 \mathrm{mH}_{2} \mathrm{O}$ de pressão de borbulhamento, e câmara de Richards. O solo foi acondicionado em anéis de $36 \mathrm{~mm}$ de diâmetro e $25 \mathrm{~mm}$ de altura e submetido a tensões entre 0,01 e $0,81 \mathrm{mH}_{2} \mathrm{O}$ na mesa de tensão, e entre 1,0 e $150 \mathrm{mH}_{2} \mathrm{O}$ na câmara de Richards. Para cada tensão foram utilizadas seis repetições. Os valores de umidade com base na massa seca (U) foram convertidos à base de volume $(\theta)\left(\theta=U . \rho_{s} / \rho_{\text {água }}\right.$, onde $\rho_{\mathrm{s}}$ e $\rho_{\text {água }}$ são, respectivamente, a massa específica do solo seco e da água) e, posteriormente, ajustados ao modelo de Van Genuchten (8), pela equação (1). Foi utilizado um programa de regressão não linear para ajustar os resultados experimentais à equação (1), conforme Boratto (1). Desse ajuste, isto é, com os parâmetros independentes $\alpha, \theta_{s}, \theta_{r}$, n e m conhecidos, além de $K_{s}$, a função $K(\theta)$ foi obtida por meio da equação (2). A condutividade hidráulica saturada $\left(\mathrm{K}_{\mathrm{s}}\right)$ foi obtida mediante aplicação da equação de Darcy (2) nas amostras acondicionadas nos anéis anteriormente mencionados.

Nos experimentos de infiltração foram obtidos oito perfis de umidade, correspondentes às seguintes situações: a) três colunas de material homogêneo de Areia Marinha nos tempos de infiltração de 5,5; 10 e 22 minutos; b) três colunas de material homogêneo de Latossolo nos tempos de 6,5; 14,5 e 17,5 minutos; e c) duas colunas estratificadas (com camadas alternadas de ambos os materiais) nos tempos de 18 e 22,5 minutos, a fim de se poder avaliar a versatilidade do modelo numérico frente a esses casos de estratificação e sua exeqüibilidade frente às situações reais de campo. Esses perfis experimentais de umidade foram comparados com os respectivos perfis obtidos por solução numérica, através do programa 1.

\section{RESULTADOS E DISCUSSÃO}

\section{Propriedades hidráulicas dos meios porosos uti- lizados}

A Tabela 1 reúne os valores de condutividade hidráulica saturada e dos parâmetros independentes obtidos por ajuste do modelo de Van Genuchten (9) aos valores experimentais da curva de retenção. A Figura 1 mostra os pontos experimentais e a curva de retenção ajustada por esse modelo para o Latossolo, e a Figura 2 para a Areia Marinha (colunas homogêneas) são mostrados nas Figuras 3, 4 e 5, respectivamente para os tempos de 5,$5 ; 10$ e $22 \mathrm{mi}-$ nutos, enquanto que os resultados para o Latossolo são mostrados nas Figuras 6,7 e 8, respectivamente para os tempos de 6,5; 14,5 e 17,5 minutos. As Figuras 9 e 10 mostram os resultados simulados e observados para os tempos de infiltração de 22,5 e 18 minutos, respectivamente, em colunas estratificadas. Pode-se observar que, de modo geral, os resultados são bastante satisfatórios em todos os casos simulados. 
PREVEDELLO, C.L. et al. Solução numérica para o processo...

TABELA 1 - Valores de Condutividade hidráulica e dos parâmetros independentes do modelo de Van Genuchten (9) para a curva de retenção.

\begin{tabular}{lrccccc}
\hline Material Poroso & $\begin{array}{r}\mathrm{Ks} \\
\mathrm{m} \cdot \mathrm{s}^{-1}\end{array}$ & $\begin{array}{c}\alpha \\
\mathrm{m}^{-1}\end{array}$ & \multicolumn{2}{c}{$\begin{array}{c}\theta_{\mathrm{s}} \\
\mathrm{m}^{3} \cdot \mathrm{m}^{-3}\end{array}$} & $\mathrm{~m}^{3} \cdot \mathrm{m}^{-3}$ & $\mathrm{r}^{2}$ \\
\hline Latossolo & 0,00035 & 28,94 & 1,32281 & 0,6266 & 0,19667 & 0,9954 \\
\hline Areia Marinha & 0,00030 & 2,52 & 4,846681 & 0,3870 & 0,01870 & 0,9883 \\
\hline
\end{tabular}

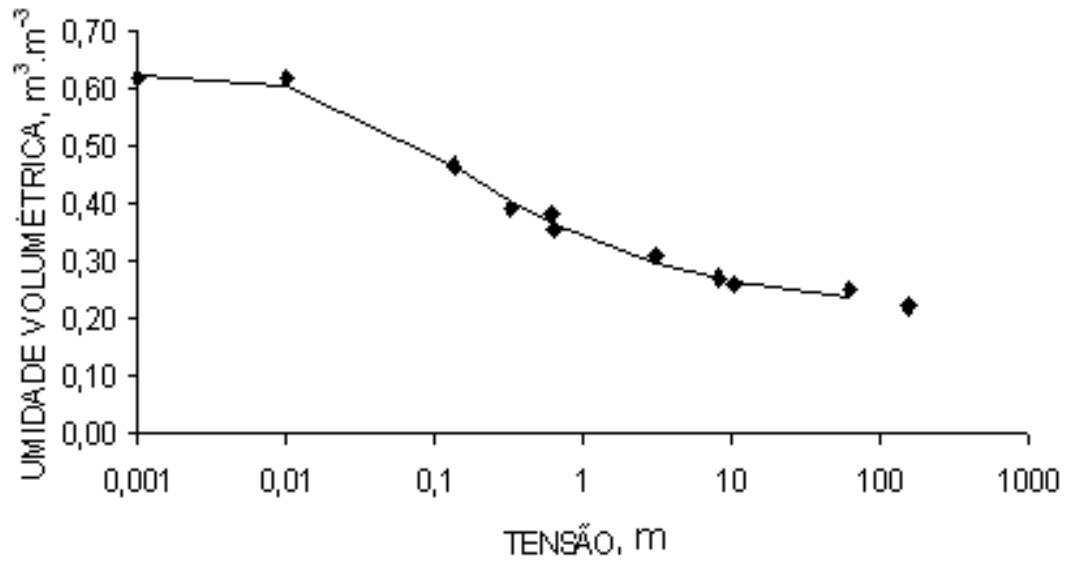

FIGURA 1 - Curva de retenção de água do Latossolo. Pontos representam valores experimentais. Linha representa o ajuste pelo modelo de Van Genuchten (9).

Como se observa, o modelo de Van Genuchten (9) se ajustou bem aos valores experimentais dos dois meios porosos, com coeficiente de determinação acima de 0,988 .

Os valores da Tabela 1 foram então introduzi- dos no programa 1 para simulação do processo da infiltração vertical, para cada um dos meios porosos estudados. Os resultados simulados e observados da infiltração na Areia Marinha.

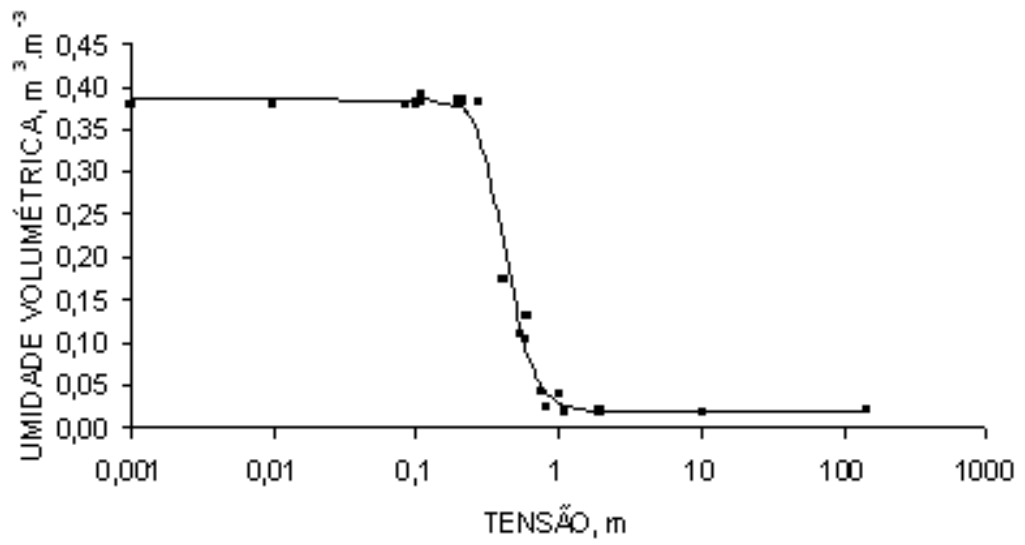

FIGURA 2 - Curva de retenção de água da Areia Marinha. Pontos representam valores experimentais. Linha representa o ajuste pelo modelo de Van Genuchten (9). 
Os maiores desvios entre os valores simulados e observados ocorreram na Areia Marinha para o tempo de 5,5 minutos (Figura 3 ), e isso pode estar associado à simplificação admitida no desenvolvimento teórico do modelo numérico (ver observação no final do item 5.2), quando se eliminou o fator unitário do denominador do modelo de Van Genuchten (9) (equação 8), prejudicando os resultados obtidos pelo modelo para tempos curtos da infiltração, ou melhor, em situações que não cumprem a condição |p/ $\rho g \mid>>1 / \alpha$. De fato, comparativamente, os desvios ocorridos no Latossolo, para o tempo de 6,5 minutos (Figura 6), foram menos importantes do que aqueles encontrados na Areia Marinha (Figura 3), uma vez que o valor de $\alpha$ do Latossolo supera o da Areia Marinha em mais de 10 vezes. Os valores estimados e observados nas diversas profundidades e tempos de infiltração, de ambos os meios porosos, foram comparados estatisticamente pelo método "Qui-quadrado" do programa Excel da Mycrosoft, e se constatou haver uma ótima aderência entre esses valores ao nível de $5 \%$ de significância.

UMIDADE VO LUMÉTRICA, m $\mathrm{m}^{-3}$

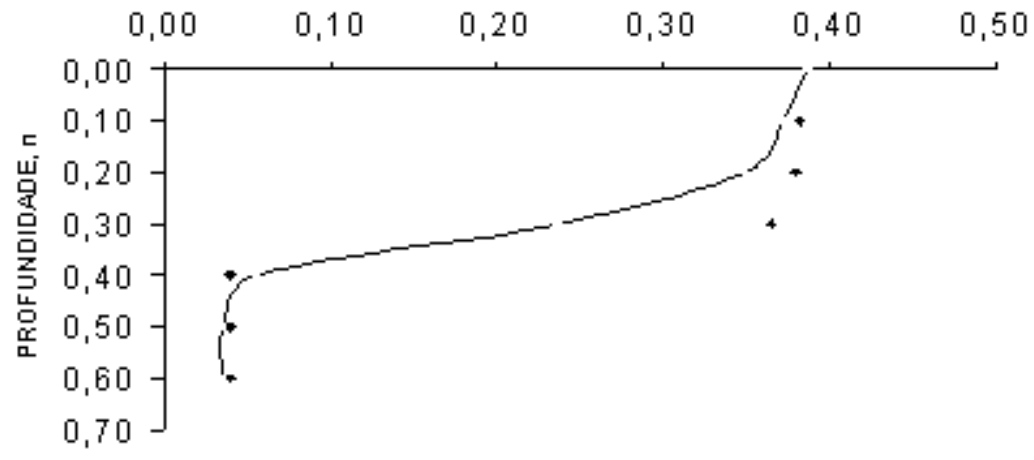

FIGURA 3 - Perfil de umidade simulado (linha) e experimental (pontos) para o processo da infiltração vertical na Areia Marinha no tempo de 5,5 minutos de infiltração.

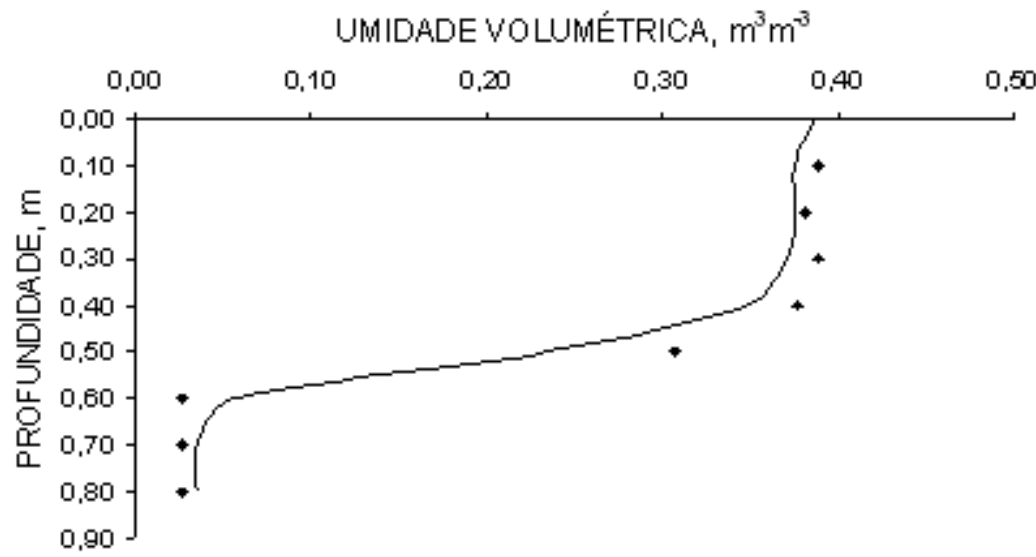

FIGURA 4 - Perfil de umidade simulado (linha) e experimental (pontos) para o processo da infiltração vertical na Areia Marinha no tempo de 10 minutos de infiltração. 
PREVEDELLO, C.L. et al. Solução numérica para o processo...

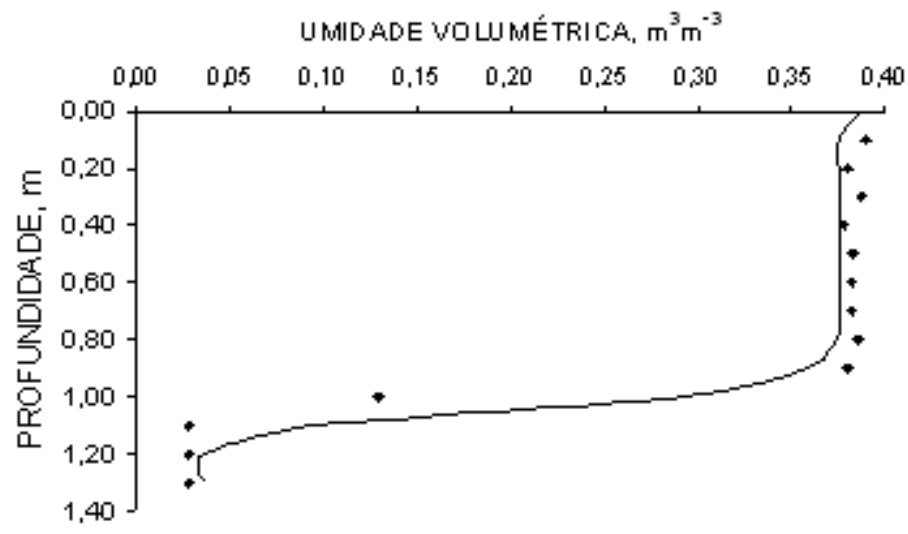

FIGURA 5 - Perfil de umidade simulado (linha) e experimental (pontos) para o processo da infiltração vertical na Areia Marinha no tempo de 22 minutos de infiltração.

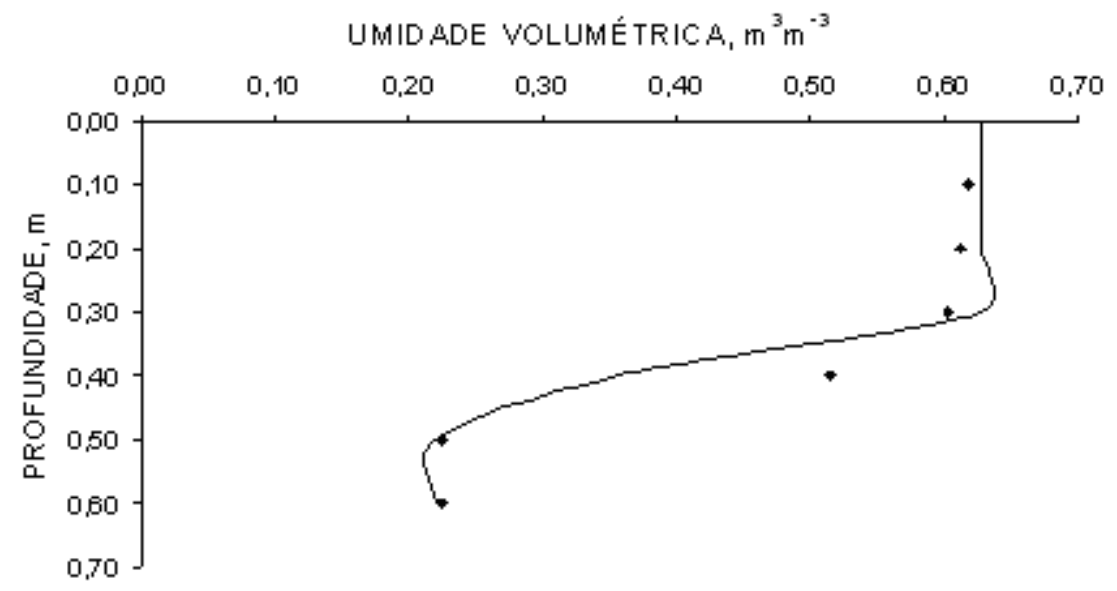

FIGURA 6 - Perfil de umidade simulado (linha) e experimental (pontos) para o processo da infiltração vertical no Latossolo no tempo de 6,5 minutos de infiltração. 


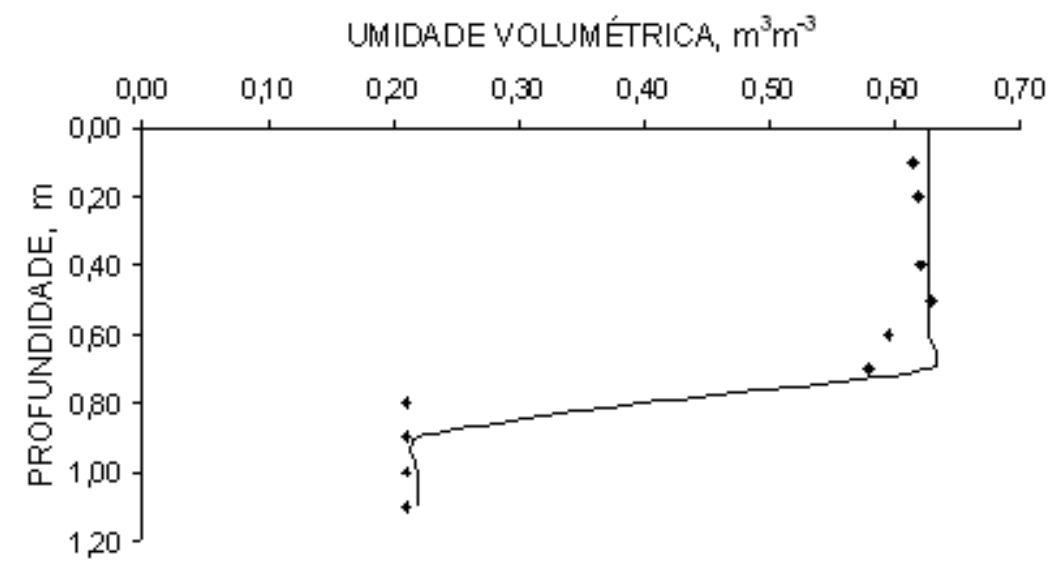

FIGURA 7 - Perfil de umidade simulado (linha) e experimental (pontos) para o processo da infiltração vertical no Latossolo no tempo de 14,5 minutos de infiltração.

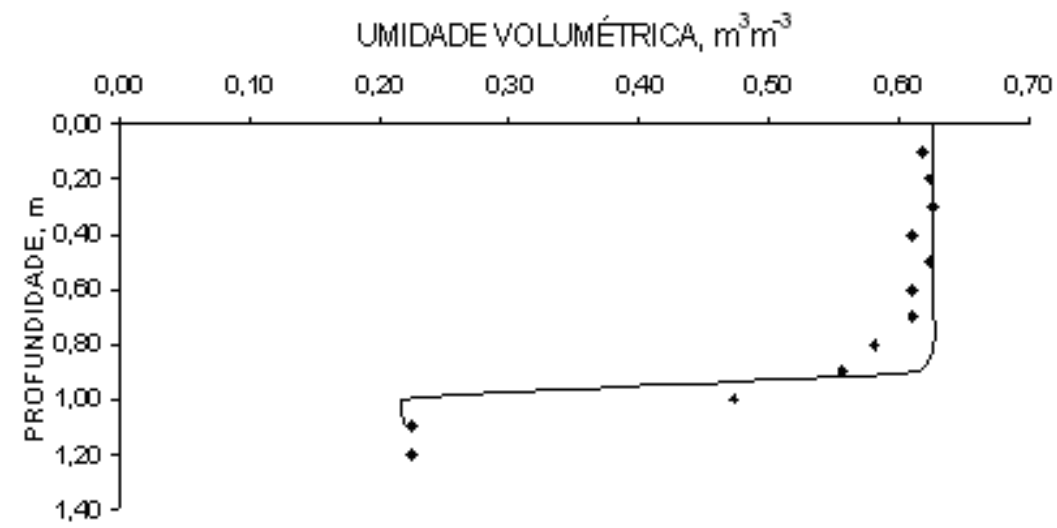

FIGURA 8 - Perfil de umidade simulado (linha) e experimental (pontos) para o processo da infiltração vertical no Latossolo no tempo de 17,5 minutos de infiltração. 
PREVEDELLO, C.L. et al. Solução numérica para o processo...

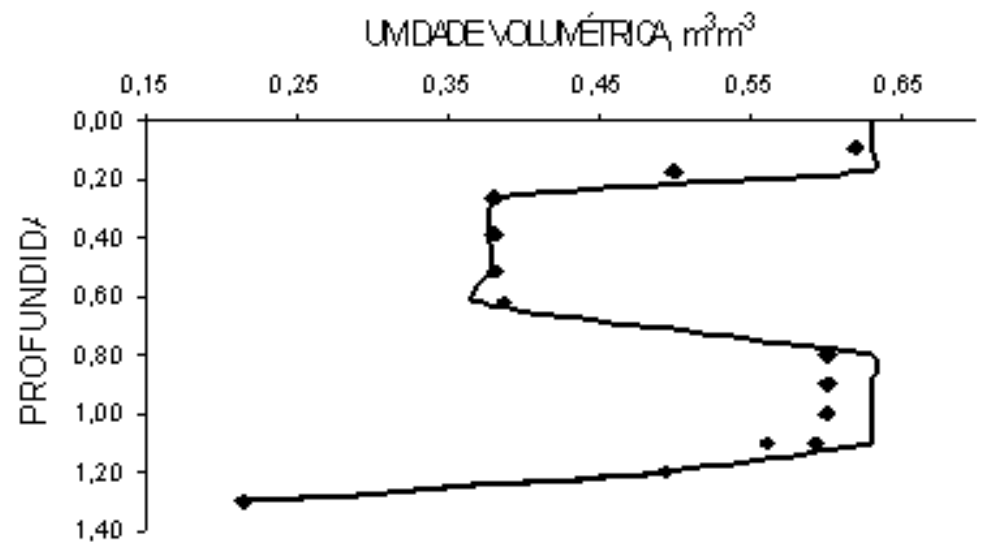

FIGURA 9 - Perfil de umidade simulado (linha) e experimental (pontos) para o processo da infiltração numa coluna estratificada com 22,5 minutos de infiltração.

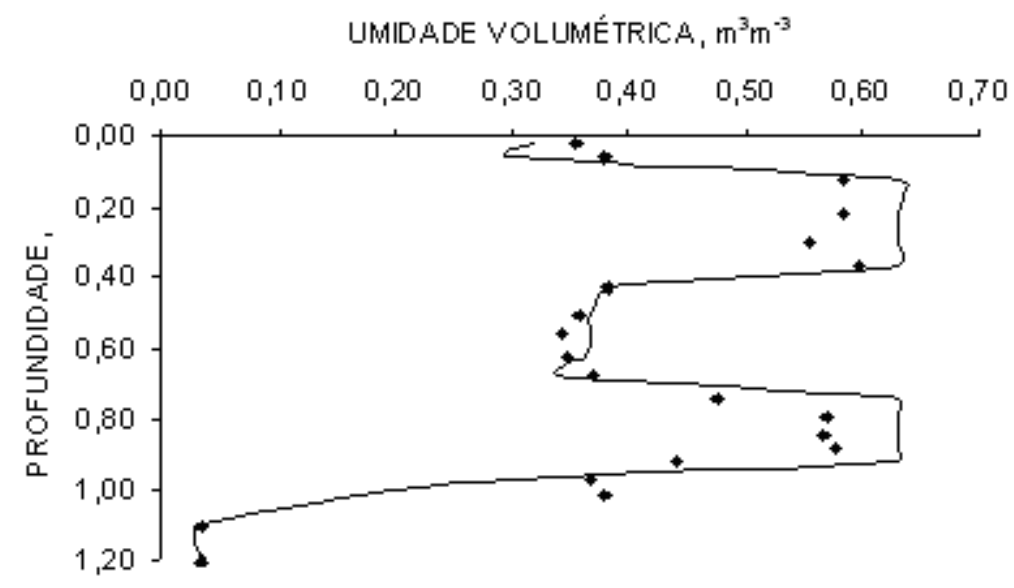

FIGURA 10 - Perfil de umidade simulado (linha) e experimental (pontos) para o processo da infiltração numa coluna estratificada com 18 minutos de infiltração. 
A Figura 9 mostra o perfil de umidade para o tempo de $22,5 \mathrm{~min}$ de infiltração numa coluna estratificada constituída de 3 camadas: a primeira, de material argiloso, da superfície até os primeiros $210 \mathrm{~mm}$; a segunda, de areia, entre 210 e $645 \mathrm{~mm}$; e a terceira, novamente de argila, entre 645 e $1200 \mathrm{~mm}$.

O mesmo procedimento foi testado, mas invertendo-se a posição dos materiais porosos na coluna e aumentando-se o número de camadas. O perfil de umidade mostrado na Figura 10 é para o tempo de 18 min de infiltração numa coluna com as seguintes camadas: a primeira, de areia, da superfície até os primeiros $90 \mathrm{~mm}$; a segunda, de argila, entre $90 \mathrm{e}$ $405 \mathrm{~mm}$; a terceira, de areia, entre 405 e $715 \mathrm{~mm}$, a quarta, de argila, entre 715 e $935 \mathrm{~mm}$; e, finalmente, a quinta, de areia, entre 935 e $120 \mathrm{~mm}$. Como se nota, o modelo se aproximou muito bem dos valores expe- rimentais, independentemente da ordem das camadas nas colunas. Também aqui, o teste estatístico "Qui-quadrado" revelou haver ótima aderência entre os valores simulados e observados, para o nível de $5 \%$ de significância.

\section{CONCLUSÃO}

A teoria proposta, resumida na forma das equações (3) e (4) ou do programa 1, é capaz de simular o processo unidimensional da infiltração vertical da água no solo, tanto para solo homogêneo quanto estratificado.

\section{REFERÊNCIAS}

1 BORATTO, F. BASIC para engenheiros e cientistas. 2 ed. Rio de Janeiro, Livros Técnicos e Científicos, 120p., 1984. DARCY, H. Les Fontaines publiques de la Ville de Dijon. Paris, Victor Dalmont, 1856.

KIRKHAM, D. \& POWERS, W. L. Advanced soil physics. 1 ed. New York, Wiley Interscience, 534 p., 1972.

MARION, J. M.; OR, D.; \& ROLSTON, D. E. Evaluation of methods for determining soil-water retentivity and unsaturated hydraulic conductivity. Soil. Sci., 18:1-13, 1994.

5 MUALEM, Y. A new model for predicting the hydraulic conductivity of unsaturated porous media. Water Resour. Res., 12:513-522, 1976.]

6 PREVEDELLO, C. L.; KOBIYAMA, M.; JACOBS, G. A. \& DIVARDIN, C. R. Comparação dos métodos do perfil instantâneo e de Van Genuchten na obtenção da condutividade hidráulica de uma areia marinha. R. bras. Ci. Solo, 19:1-5, 1995.

7 PREVEDELLO, C. L. Física do solo com problemas resolvidos. 1 ed. Curitiba, Salesward-Discovery, 446p., 1996.

8 SIMUNEK, J. VAN GENUCHTEN, M. T.; GRIBB, M. M. \& HOPMANS, J. Parameter estimation of unsaturated soil hydraulic properties from transient flow processes. Soil Tillage Res., 47:27-36, 1998.

9 VAN GENUCHTEN, M. Th. A closed-form equation for predicting the hidraulic conductivity of unsaturated soils. Soil Sci. Soc. Am. J., 44:892-898, 1980. 